\title{
An improved method of MRI segmentation based on level set
}

\author{
Zuojun Liu, ${ }^{1, a}$, Lihong $\mathrm{Li}^{2, \mathrm{~b}}$ \\ ${ }^{1}$ Faculty of Computer and Software Engineering, Huaiyin Institute of Technology, Huai'an, Jiangsu, \\ China \\ ${ }^{2}$ Faculty of Foreign Language, Huaiyin Institute of Technology, Huai'an, Jiangsu, China \\ ahyliuzj@126.com, bhylilihong@126.com
}

\begin{abstract}
Keywords: MRI segmentation; level set; internal energy; re-initialization; external energy
Abstract: In traditional method of MRI segmentation based on level set, there is a question that the level set function deeds periodical re-initialization which will cost lots of times. In order to solve this problem, a simple internal energy is introduced in the energy function to eliminate the cost of periodical re-initialization. On the other hand, an external energy is introduced to get better robust in segmentation of noisy MRI. Proved by lots of experiments, the method proposed in this paper is efficient in solving the question of re-initialization with better robustness.
\end{abstract}

\section{Introduction}

The image segmentation is the basis of image analysis and computer vision. In recent years, all kinds of methods had been proposed about this problem. In all of these methods, geometric active contour method is widely used in solving this problem ${ }^{[1,2,3]}$.

The $2 \mathrm{D}$ curve is expressed as a zero level set of $3 \mathrm{D}$ continuous function $\mathrm{z}=\square(\mathrm{x}, \mathrm{y}, \mathrm{z})$ in the traditional method of geometric active contour. The level set function $\square(\mathrm{x}, \mathrm{y}, \mathrm{z})$ is defined as a symbolic distance functions. The curve evolution based on level set update the level set function continuously to evolve the zero level set. The merits of this method are as follow: (1) The geometric active contour can combine and fracture automatically which can deal with the change of topology; (2) The level set function is a simple function which can be compute with simple method.

The geometric active contours methods which get the evolution by minimize an energy function are based on the level set. The methods of geometric active contour based on level set can introduce more information in the energy function compared to the traditional method of geometric active contour, for example, the region information and the intensity information. So, the methods of geometric active contour based on level set are adaptive with higher segmentation quality.

The methods of geometric active contour should keep the level set function approximating a closed symbol distance function. the level set should be re-initiative realty to make sure that the level set function approaches to the symbol distance function. So, some questions occur: (1) the repeat re-initializations will cause lots of computation. (2) Lots of time for re-initialization.

In this paper, a simple internal energy is introduced in the energy function to eliminate the cost of periodical re-initialization and an external energy is introduced to get better robustness in the segmentation of noisy MRI.

\section{The method of geometric active contour without re-initialization}

In document [4], a method based on geometric active contour is proposed. The advantage of this method is that the level set function need not to be re-initialized in evolving by introducing a new energy function. The energy function includes two parts: (1) Internal energy, which is called internal constraint energy in this paper. It is only relevant to the level set function which constraints the level set function approaches a symbol distance function. (2) External energy. The main function of external energy is to drive the zero level set to move toward the edge of an object. The internal energy in this document is defined as formula (1),

$$
\mathrm{P}(\emptyset)=\int_{\Omega} 1 / 2(|\phi|-1)^{2} d x d y
$$

In this formula, $\Omega$ is the region of the image. $\mathrm{P}(\Phi)$ is the a measurement of the level set 
function deviating the symbol distance function. During the process of minimization, $|\nabla \emptyset|$ approaches 1 . So, the level set function will approximate the symbol distance function in evolving.

The energy function with internal constraint energy is shown as formula (2),

$$
\begin{aligned}
& \mathrm{E}(\Phi)=\mu \mathrm{P}(\Phi)+\mathrm{E}_{\mathrm{g}}(\Phi)=\mu \mathrm{P}(\Phi)+\lambda \mathrm{L}_{\mathrm{g}}(\Phi)+\mathrm{vA}_{\mathrm{g}}(\Phi) \\
& =\frac{1}{2} \mu \int_{\Omega}(|\varnothing|-1)^{2} \mathrm{dxdy}+\lambda \int_{\Omega} \mathrm{g}\left(\mathrm{I}_{\sigma}\right) \delta(\Phi)|\nabla \emptyset| \mathrm{d} x \mathrm{dy}+\mathrm{v} \int_{\Omega} \mathrm{g}\left(\mathrm{I}_{\sigma}\right)(\varnothing) \mathrm{H}(-\emptyset) \mathrm{dxdy}
\end{aligned}
$$

In formula (2), $\mu, \lambda>0 . v$ is a constant to control the direction of level set. $\delta$ is Dirac function. $\mathrm{H}$ is Heaviside function. $\mathrm{G}_{\mathrm{g}}$ is Gauss function. $\nabla$ is gradient operator. $\mathrm{E}_{\mathrm{g}}(\varnothing)$ is the external energy which drive the level set to move toward the edge of the object. $g\left(\mathrm{I}_{\sigma}\right)=\frac{1}{1+\left|v G_{\mathrm{g}} *\right|^{\mathbb{F}}}, \mathrm{P}=1,2$, is the stopping function. $\mathrm{L}_{\mathrm{g}}(\phi)=\int_{\Omega} \mathrm{g}\left(\mathrm{I}_{\sigma}\right) \delta(\phi)|\nabla \phi| \mathrm{dxdy}$ is the weighted arc length to make the curve of zero level set to be smooth. $A_{g}(\phi)=\int_{\Omega} g\left(I_{\sigma}\right)(\varnothing) H(-\emptyset) \mathrm{d} x d y$, is the weighted area. If the $v>0$, the curve of the zero level set shrinkages toward inside. If the $v<0$, the curve of the zero level set extends toward outside.

The minimum value of formula (2) is equivalent to the solution of the following equation,

$$
\frac{\partial \phi}{\partial \mathrm{t}}=\mu\left(\Delta \emptyset-\operatorname{div}\left(\frac{\nabla \phi}{|\nabla \phi|}\right)\right)+\lambda \delta(\phi) \operatorname{div}\left(\mathrm{g}\left(\mathbf{I}_{\sigma}\right) \frac{\nabla \phi}{|\nabla \phi|}\right)+v \delta(\phi) \mathrm{g}\left(\mathrm{I}_{\sigma}\right)
$$

The initiative condition is $\emptyset(\mathrm{x}, \mathrm{y}, 0)=\emptyset_{0}(x, y)$. The image segmentation with above equation can get some advantages: (1) the level set function needs not to be re-initialized. (2) the step can be increased to accelerate convergence. (3) the initiative level set function need not to be defined as the symbol distance function. The following formula is adopted as the initiative level set function in document [4],

$$
\emptyset_{0}(x, y)=\left\{\begin{array}{c}
-\rho(x, y) \in \Omega^{-} \\
\rho(x, y) \in \Omega^{+}
\end{array}\right.
$$

In this paper, a new improved segmentation method based on level set according document [4] is proposed. The advantages of this method are as follows: (1) this method is fast in deal with clean or low noisy image. (2) the level set function can pass the noisy region quickly and reach the edge of the image.

\section{New method of geometric active contour without re-initialization}

\section{The internal constraint energy}

The function of internal energy $\mathrm{P}(\Phi)$ make sure that the level set function is just the symbol distance function during evolving.

$$
\frac{\partial \phi}{\partial \mathrm{t}}=\Delta \emptyset-\operatorname{div}\left(\frac{\nabla \emptyset}{|\nabla \sigma|}\right)=\operatorname{div}\left(\left(1-\frac{1}{\nabla \phi}\right) \nabla \emptyset\right)
$$

The formula (5) is a heterosexual spread model with speed $1-1 / \nabla \emptyset$. If $\nabla \emptyset>1$, the formula (5) shows forward diffusion. If $\nabla \emptyset<1$, the formula (5) shows revise diffusion. So, the function of formula (5) is to insure that the gradient value satisfies $|\nabla \emptyset| \approx 1$. So, the level set function can't be too steep or too plan.

An internal constraint energy is added into formula (3). If the initiative function is not the symbol distance function, the level set function can't completely approach the symbol distance function in the region of the whole image during evolution. But, near the zero level set, the level set function is approximate to the symbol distance function. The internal constraint energy can make the level set function to be smooth near the zero level set. So, a simple internal constraint energy is introduced,

$$
\mathrm{P}(\varnothing)=\frac{1}{2} \int_{\Omega}(|\nabla \phi|)^{2} d x d y
$$

$\mathrm{P}(\Phi)$ describes the smoothness of level set function in the whole region of an image. The level set function will become sooth during minimization. Proved by lots of experiments, this internal energy is much more efficient than that of document [4]. 


\section{External energy}

In document [4], $\mathrm{g}\left(\mathrm{I}_{\sigma}\right)=\frac{1}{1+\left|\nabla G_{\sigma} \mathrm{k}\right|^{P}}, \mathrm{P}=1,2$, is stopping function. $\mathrm{g}\left(\mathrm{I}_{\sigma}\right)$ is the gradient value. $|\nabla I|$ is the reduction function of variables. In smooth region of the image, $|\nabla I|$ is small. $g\left(I_{\sigma}\right)$ approaches 1. The zero level set passes smooth region quickly. At the edge of the image, $|\nabla I|$ is larger. $g\left(I_{\sigma}\right)$ approaches 0 and the level set function will stop at the edge of the image. So, the segmentation of an image is completed. But, for a lonely noise, $|\nabla I|$ will be larger with smaller valve stopping function. Then, the speed for the level set function to pass the lonely noise will be slower. The number of iterations will be increased. According to these discuses above, a new edge detection operator is proposed in document [4]. $\mathrm{D}=\left\|u_{\eta \eta}|-| u_{\xi}\right\|, u_{\eta \eta}$ is a second order directional derivative of $u$ on the direction of normal. $u_{\xi \xi}$ is a second order directional derivative of $u$ on the direction of tangent. The characteristics to differentiate the different region of an image with operate D are as follows: (1) at the edge of an image, $\left|u_{\eta \eta}\right|$ is large and $\left|u_{\xi \xi}\right|$ is small. So, D is large. (2) to the lonely noise, $\left|u_{\eta \eta}\right|$ and $\left|u_{\xi \xi}\right|$ are large and D is small.

In this paper, the edge detection operator is used in stopping function. So,

$$
\mathrm{g}\left(\mathrm{I}_{\sigma}\right)=\frac{1}{1+\left|\mathrm{D}\left(G_{\sigma}+1\right)\right|^{F}} \mathrm{P}=1,2
$$

According to the definition of stopping function, in the region of noisy, D approaches 0 and $g\left(\mathrm{I}_{\sigma}\right)$ approaches 1 . These demonstrated that zero level set of level set function can pass the region with noise quickly and reach the edge of image and get the correctly segmentation results.

\section{The energy function}

In this paper, a new stopping function (7) is introduced into the external energy. The final energy function is defined as follow,

$$
\begin{aligned}
& E(\Phi)=\mu P(\Phi)+E_{g}(\phi)=\mu P(\phi)+\lambda L_{g}(\Phi)+v A_{g}(\Phi) \\
& =\frac{1}{2} \int_{\Omega}(|\nabla \Phi|)^{2}+\lambda \int_{\Omega} g\left(I_{\sigma}\right) \delta(\Phi)|\nabla \Phi| d x d y+\mathrm{v} \int_{\Omega} g\left(I_{\sigma}\right) \mathrm{H}(-\Phi) d x d y
\end{aligned}
$$

The zero level set moves toward the edge of the image drived by external energy. At last, the zero level set function will stop at the edge of the image. The internal energy keeps the level set function to be smooth during evolution. In this paper, Heaviside function and Dirac function are used.

$H_{z}(x)=\frac{1}{2}\left(1+\frac{2}{\pi}\right) \arctan \left(\frac{x}{s}\right), \delta_{s}(x)=H_{s}(x)$ when the value of $\varepsilon^{->0}, H_{s}$ and $\delta_{z}$ will approach the standard Heaviside function and Dirac function.

The minimum value got by method of gradient descent is equivalent to the solution of the following equation.

$$
\frac{\partial \emptyset}{\partial \mathrm{t}}=\mu \Delta \emptyset+\lambda \delta_{s}(\phi) \operatorname{div}\left(g\left(I_{\sigma}\right) \frac{\eta \emptyset}{|v \phi|}\right)+v \delta_{\varepsilon}(\phi) g\left(I_{\sigma}\right)
$$

The initiative condition: $\phi(x, y, 0)=\emptyset_{0}(x, y)$. The first item is corresponding to the gradient flow of the internal constraint energy. The second item is corresponding to the gradient flow of $\mathrm{L}_{\mathrm{g}}(\emptyset)$. The second third item is corresponding to the gradient flow of $\mathrm{A}_{\mathrm{g}}(\emptyset)$.

\section{Experimental verification}

The validity of the method proposed in this paper is verified through lots of experiments. These experiments proved: (1) the method need not re-initiation during iterations. (2) This method gets excellent results at time cost and segmentation quality.

The experiment platform: Windows 8, I5 CPU, 4GB, Mat lab 2016a. The parameters are as following: $\varepsilon=1.6, \mu=0.05, \lambda=6, v=1.5, P=2, \Delta x=\Delta y=1, \Delta t=4$. The method proposed in this paper is mainly applied in MRI segmentation. These images are all selected from two databases of MRI, Caltech101and Caltech256.In order to verify the method proposed in this paper, the results of this 
paper and document [4] are shown in table 1 and table 2. Table 1 shows the segmentation results. Table 2 shows the times of the two methods.

Table 1 experimental results

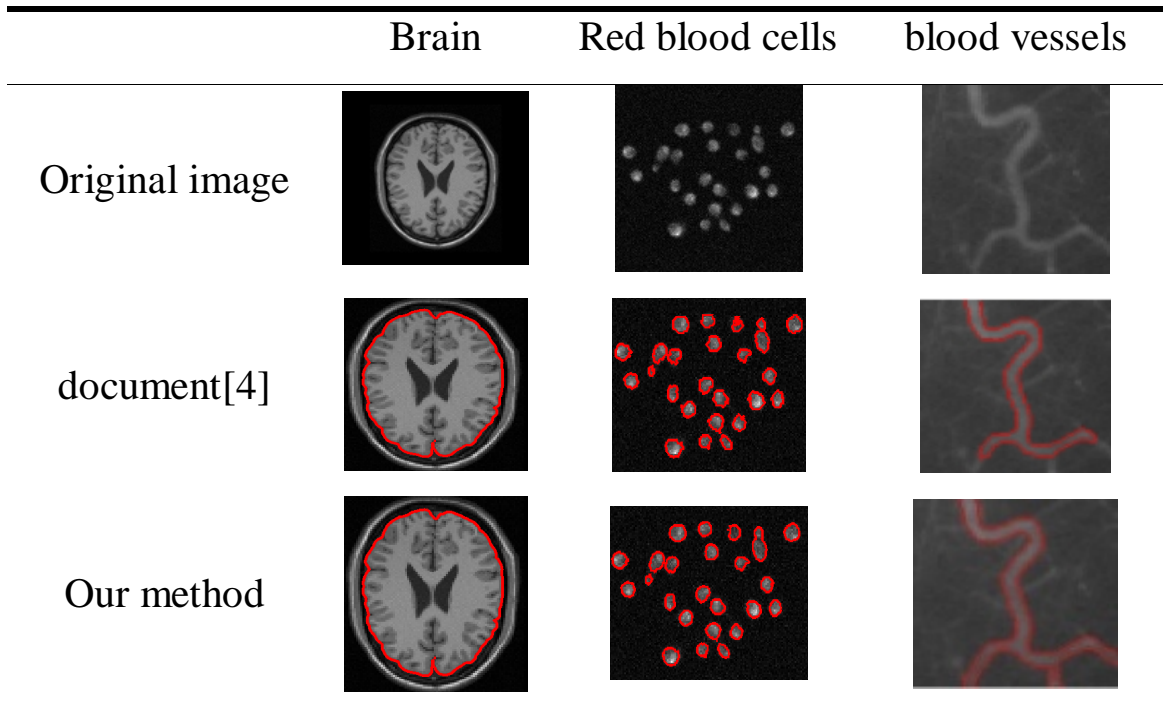

The experiments are carried out at the same conditions, including hardware and software. The images used in the experiments are MRI, including: red blood cells, brains and blood vessels. Seen from the experimental results, the method proposed in this paper is more accurate than the method in document [4]. To the first image, the results of two methods are similar. To the second image, the method in document [4] is less accurate. There are some errors. To the third image, the segmentation in document [4] is not complete.

Table 2 times comparisons

\begin{tabular}{cccc}
\hline & Brain & Red blood cells & blood vessels \\
\hline Document [4] & 2.42 & 3.14 & 3.11 \\
Our method & 2.10 & 2.99 & 2.97 \\
\hline
\end{tabular}

Seen from table 2, after improvement, the method in this paper is more efficient.

\section{conclusions}

After an analysis to the method in document [4], an improved MRI segmentation method based on level set is proposed. This method can avoid the problem of re-initiation. The effect and efficiency is more better than the method in document [4].

\section{References}

[1]Caselles V, Catte F, Cull T.A geometric model for active contours in image processing[J] . Numerische Mathmatik, 1993, 66(1):1-31.

[2] Caselles V, Kimmel R, Sapiro G.Geodesic active contours EJ].International Journal of Computer Vision, 1997, 22:61-79 .

[3] Chan T, Vese L.Active contours without edges[J].IEEE Transactions on Image Processing, 2001, 10(2): 266-277 . 
[4] Li Chunming, Xu Chenyang, Gui Changfeng.level set evolution without re-initialization:A new variational formulation[c]//2005 IEEE Conferenceon Computer Vision and Pattern Recognition.[S.1.]:IEEE Computer Society Press, 2005:430-436 . 